\title{
Prediction of airblast loads in complex environments using artificial neural networks
}

\author{
A. M. Remennikov ${ }^{1}$ \& P. A. Mendis ${ }^{2}$ \\ ${ }^{1}$ School of Civil, Mining and Environmental Engineering, \\ University of Wollongong, Australia \\ ${ }^{2}$ Department of Civil and Environmental Engineering, \\ The University of Melbourne, Australia
}

\begin{abstract}
Predicting non-ideal airblast loads is presently a complex computational art requiring many hours of high-performance computing to evaluate a single blast scenario. The goal of this research is to develop a method for predicting blast loads in a non-ideal environment in real time. The proposed method is incorporated in a fast-running model for rapid assessment of blast loads in complex configurations such as a dense urban environment or a blast environment behind a blast barrier.

This paper is concerned with an accurate prediction of the blast loads from a bomb detonation using a neural network-based model. The approach is demonstrated in application to the problem of predicting the blast loads in city streets. To train and validate the neural networks, a database of blast effects was developed using the Computational Fluid Dynamics (CFD) blast simulations. The blast threat scenarios and the principal parameters describing the street configurations and the blast wall geometry were used as the training input data. The peak pressures and impulses were used as the outputs in the neural network configuration.
\end{abstract}

Keywords: neural networks, blast loads, urban environment, explosion, numerical simulation.

\section{Introduction}

Protecting civilian buildings from the threat of terrorist activities is one of the most critical challenges for structural engineers today. Events of the past few years have greatly heightened the awareness of structural designers of the threat 
of terrorist attacks using explosive devices. Extensive research into blast effects analysis and methods of protective design of buildings has been initiated in many countries to develop methods of protecting critical infrastructure and the built environment.

Although it is recognised that no civilian buildings can be designed to withstand any conceivable terrorist threat, it is possible to improve the performance of structural systems by better understanding the factors that contribute to a structure's blast resistance. One of such factors is the ability of the structural designer to accurately predict the blast loadings on structural components using analytical or numerical tools that take into account the complexity of the building, the presence of nearby structures, and the blast wave-structure interaction phenomena.

In recent years, the use of non-traditional tools based on artificial intelligence has received significant attention from the civil engineering researchers in relation to the systems that exhibit dynamic, multivariate, and complex behaviours (e.g. wave forces, weather conditions, shock and impact problems). In this paper, an approach based on the neural network methodology is developed to train the neural networks capable of predicting the blast resultants in the complex geometries with reasonable accuracy, cost, and minimum computing requirements. For brevity, the approach is demonstrated only in application to the problem of predicting the blast loads in city streets. The approach has also proven to be effective in other complex blast wave-structure interaction problems such as predicting the blast environment behind a rigid blast barrier.

\section{Effect of adjacent structures on blast loads on buildings}

Blast loads in simple geometries can be predicted using empirical or semi-empirical methods. These can be used to calculate blast wave parameters for hemispherical or spherical explosive charges detonated near the surface or in a free air to predict blast effects on isolated structures and structural components.

Events of the recent years have demonstrated that the most common source of unplanned explosions were terrorist devices in urban environment. In complex urban geometries, the blast wave behaviour can only be predicted from first principles using such numerical tools as AUTODYN [1], Air3D [2], and some others. Such tools solve the governing fluid dynamics equations and can be used to simulate three-dimensional blast wave propagation including multiple reflections, rarefaction and diffraction. In addition, Computational Fluid Dynamics (CFD) techniques can capture such key effects as blast focussing due to the level of confinement, shielding by other buildings and component failure (e.g. a window failure).

\section{Neural networks}

Artificial neural networks (ANNs) are computational models loosely inspired by the neuron architecture and operation of the human brain [3]. They are massively 
parallel; they can process not only clean but also noisy or incomplete data. ANNs can be used for the mapping of input to output data without knowing 'a priori' a relationship between those data. ANNs can be applied in optimum design, classification and prediction problems.

An artificial neural network is an assembly (network) of a large number of highly connected processing units, the so-called nodes or neurons. The neurons are connected by unidirectional communication channels ("connections"). The strength of the connections between the nodes is represented by numerical values, which normally are called weights. Knowledge is stored in the form of a collection of weights. Each node has an activation value that is a function of the sum of inputs received from other nodes through the weighted connections.

The neural networks are capable of self-organisation and knowledge acquisition, i.e. learning. One of the characteristics of neural networks is the capability of producing correct, or nearby correct, outputs when presented with partially incomplete inputs. Further, neural networks are capable of performing an amount of generalization from the patterns on which they are trained. Most neural networks have some sort of "training" rule whereby the weight of connections is adjusted on the basis of presented patterns. Training consists of providing a set of known input-output pairs, patterns, to the network. The network iteratively adjusts the weights of each of the nodes so as to obtain the desired outputs within a requested level of accuracy. Error is defined as a measure of the difference between the computed pattern and the expected output pattern.

\subsection{Multilayer Perceptron Network (MLP)}

The multi-layer perceptron (MLP) network trained by means of the back-propagating algorithm is currently given the most attention by application developers. The MLP network belongs to the class of layered feed-forward nets with supervised learning. A multi-layer neural network is made up of one or more hidden layers placed between the input and output layers as shown in Figure 1.

Each layer consists of a number of nodes connected in the structure of a layered network. The typical architecture is fully interconnected, i.e. each node in a lower level is connected to every node in the higher level. Output units cannot receive signals directly from the input layer. During the training phase activation flows are only allowed in one direction, a feed-forward process, from the input layer to the output layer through the hidden layers. The input vector feeds each of the first hidden layer nodes, the outputs of this layer feed into each of the second hidden layer nodes and so on.

At the start of the training process the weights of the connections are initialised by random values. During the training phase, representative examples of input-output patterns are presented to the network. Each presentation is followed by small adjustments of weights and thresholds if the computed output is not correct. If there is any systematic relationship between input and output and the training examples are representative of this, and if the network topology is properly chosen, then the trained network will often be able to generalize 
beyond learned examples. Generalization is a measure of how well the network performs on the actual problem once training is complete. It is usually tested by evaluating the performance of the network on new data outside the training set.

Generalization is most heavily influenced by three parameters: the number of data samples, the complexity of the underlying problem and the network architecture. Currently, there are no reliable rules for determining the capacity of a feed-forward multi-layer neural network. Generally, the capacity of a neural network is a function of the number of hidden layers, the number of processing units in each layer, and the pattern of connectivity between layers.

During first stage of creating an artificial neural network to model an input-output system is to establish the appropriate values of the connection weights and thresholds by using a learning algorithm. A learning algorithm is a systematic procedure for adjusting the weights and in the network to achieve a desired input - output relationship, i.e. supervised learning. The most popular and successful learning algorithm used to train multi-layer neural network is currently the back-propagation routine.

\section{Hidden Layer}

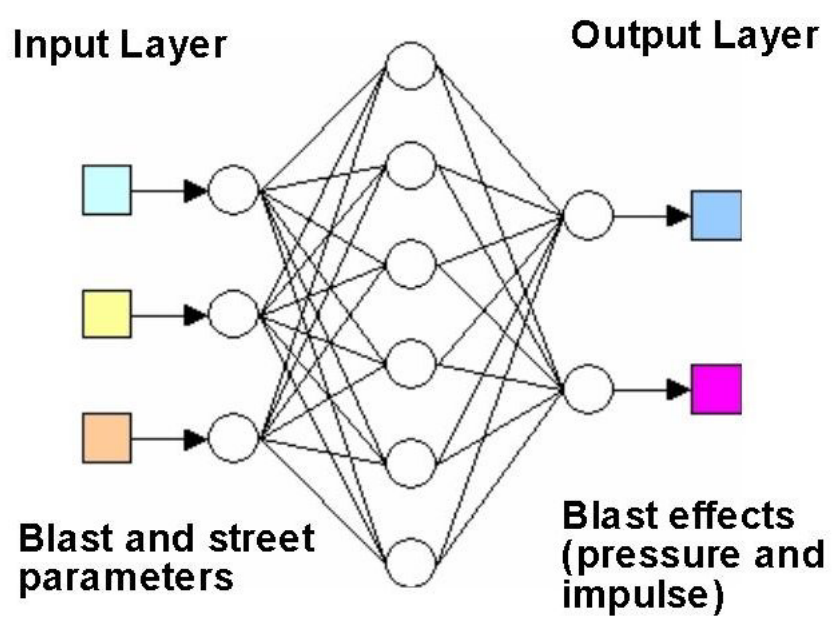

Figure 1: A simple back-propagating network for evaluation of blast effects in city streets.

\subsection{Use of neural networks for predicting blast loads}

The problem of blast in an urban environment is manifold; there are several different aspects that are of interest. Principally, among these are the direct effects of blast on people (both indoors and outdoors), the indirect effects of blast on people: from broken glazing, fallen masonry and collapsed building, and damage to buildings. 
It is important to appreciate these problems if evacuation distances or safe areas within buildings are to be identified. All the above considerations have one common feature: they can only be quantified once the precise blast environment (in terms of pressure and impulse) is known throughout the region of interest.

Unfortunately, the effect of urban geometry on the propagation of blast waves is vast and complicated subject, and only recently has it begun to be approached in fundamental and systematic manner (Rose and Smith [4], Remennikov [5, 6]) and Remennikov and Rose [7]. In this paper, investigation of the effect of the street configurations on the pressure and impulse is one of the primary objectives. In order to quantify this effect, the pressure and impulse enhancement factors are defined as:

$$
\begin{gathered}
\text { Pressure enhancement factor }\left(\mathrm{E}_{\mathrm{p}}\right)=\frac{\text { Peak pressure }_{\text {street configuraton }}}{\text { Peak pressure }_{\text {unconfined burst }}} \\
\text { Impulse enhancement factor }\left(\mathrm{E}_{\text {imp }}\right)=\frac{\text { Peak impulse }}{\text { street configuraton }} \\
\text { Peak impulse } \\
\text { unconfined burst }
\end{gathered}
$$

One of the goals of the presented study was to develop a fast-running tool for predicting blast loads in an urban environment. This was accomplished by training an artificial neural network to approximate the pressure and impulse coefficients generated by a series of Computational Fluid Dynamics (CFD) numerical simulations for a selected street configuration and by varying the principal geometrical parameters of this street configuration. This possibility arises because every pressure monitoring location on the building façade can be identified by four independent parameters:

\begin{tabular}{lc}
\hline \multicolumn{1}{c}{ Parameter } & {$\left[\mathrm{m} / \mathrm{kg}^{1 / 3}\right]$} \\
\hline scaled street width & $w / W^{1 / 3}$ \\
scaled building height & $h / W^{1 / 3}$ \\
scaled distance along the street & $x / W^{1 / 3}$ \\
scaled height of the monitoring & $y / W^{1 / 3}$ \\
location above the ground & \\
\hline
\end{tabular}

These four parameters can fully characterise the highly non-linear relationship between the explosive source weight, $W$, the standoff parameters $x$ and $y$, and the resulting peak pressure and peak scaled impulse. Therefore, these four parameters could be used as inputs to train the neural network using data generated by the CFD numerical simulations.

The problem of blast loads in an urban environment on the basis of CFD simulated numerical data is essentially a prediction (interpolation) problem. Since artificial neural networks are proving to be an effective tool for predicting values of blast loads, the basic idea in a neural network based approach is to train a network with patterns of the street and blast scenario parameters describing the spatial distribution of blast loads on building facades. This implies that each pattern represents the unique value of peak pressure and peak scaled impulse at 
each of the monitoring locations due to detonation of an explosive charge, $W$, at a particular location described by values of $x$ and $y$, in a street with principal parameters $w$ and $h$. Therefore, the patterns of the quantities describing the blast environment are used as inputs and the peak pressure and peak scaled impulse as outputs to train the neural network.

The training of a neural network with appropriate data containing the information about the cause and effect is a key requirement of a neural network approach. This means that the first step is to establish the training set, which can be used to train a network in a way that the network can predict the blast effects with the reasonable accuracy of 5 to $10 \%$ of the CFD-generated results. Ideally, the training set should contain data obtained by measurements, model tests or through numerical simulation, or through a combination of all three types of data.

In order to verify how well a trained network has learned the training cases, the trained network is tested by subjecting it to the training sets. The important generalization capability of a neural network for predicting blast wave parameters is tested by subjecting the trained network to data not included into the training sets (the so-called validating sets). How well a trained network is to generalize depends on the adequacy of the selected network architecture and the information on the blast load environment included in the training sets.

This study considers a broad range of scaled street widths: $w / W^{1 / 3}=0.8$ to 3.2 $\mathrm{m} / \mathrm{kg}^{1 / 3}$ and covers much of the range of practical interest. Similarly, scaled building heights from $h / W^{1 / 3}=0.4$ to $2.4 \mathrm{~m} / \mathrm{kg}^{1 / 3}$ is considered, which roughly equates to one to six storey buildings for a charge weight of $1000 \mathrm{~kg}$. The scaled distance along the street was limited to the range 0.0 to $10.0 \mathrm{~m} / \mathrm{kg}^{1 / 3}$, which equates to a $100-\mathrm{m}$ distance along the street for a $1000 \mathrm{~kg}$ explosive charge. Results of this study will lead to the possibility of using the information to predict blast loads (pressures and impulses) at any location in a street from knowledge of the charge weight, street width and building height.

\subsection{Preparation of the dataset}

The programme of numerical simulations was designed to cover most of the range of street widths that exist in real cities. Seven different street widths: $w=8,12,16,20,24,28$ and $32 \mathrm{~m}$ were considered. Similarly, the six different building heights: $h=4,8,12,16,20$ and $24 \mathrm{~m}$, which modelled one to six storey buildings, respectively, were used. For each street width, an additional simulation was performed for no buildings in the model. This last analysis produced side-on pressures and was used to evaluate values of the pressure and impulse enhancement factors. A total of 49 analyses were performed in this matrix to establish the enhancement factors data sets for training of the neural network.

A typical straight city street configuration is shown in Figure 2 . The distance along the street $l$, the width $w$, and the location of a hypothetical hemispherical explosive charge $W$ are indicated. Figure 2 also shows the end view of the street where the building height $h$ and the building depth $d$ are indicated. 
The parametric study was based on a $1000 \mathrm{~kg}$ TNT hemispherical charge, detonated on the centreline of the street. A computational domain $x=125 \mathrm{~m}$ (along the street) by $y=48 \mathrm{~m}$ wide and $z=48 \mathrm{~m}$ wide was used, requiring about $2,500,000$ computational cells. Pressure monitoring points were located at $5 \mathrm{~m}$ intervals from $x=0 \mathrm{~m}$ to $x=100 \mathrm{~m}$ along the length of the street and in the vertical plane at levels $\mathrm{y}=2 \mathrm{~m}, 6 \mathrm{~m}, 10 \mathrm{~m}, 14 \mathrm{~m}, 18 \mathrm{~m}$ and $22 \mathrm{~m}$ above the ground. The computational domain was extended sufficiently far in each direction to ensure that the presence of the boundaries did not affect the solutions at the pressure measuring locations.
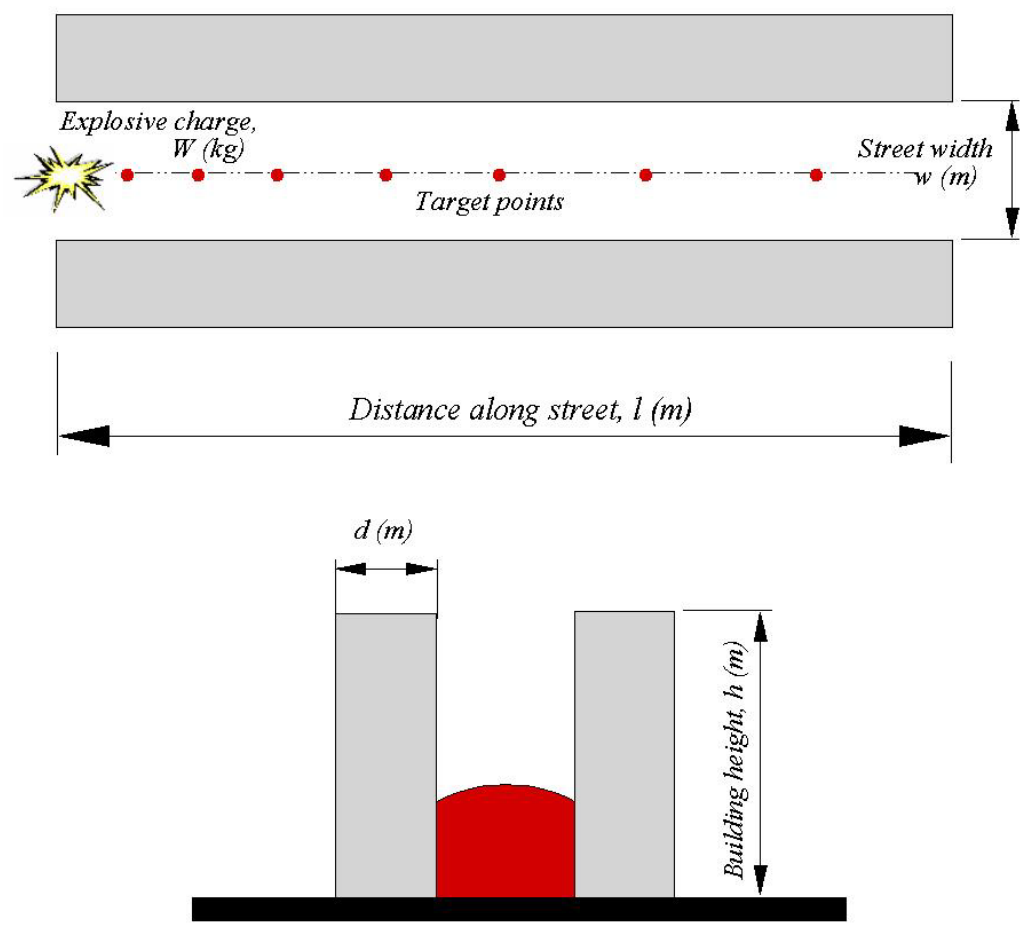

Figure 2: $\quad$ Plan and end view of a straight city street configuration.

\subsection{Neural network implementation}

The implementation stage of a neural network model typically includes the following two tasks: (1) data preparation, and (2) training and testing. Data preparation was conducted by performing a series of blast propagation analyses for the geometry shown in Figure 2 and using the test matrix of different building heights, street widths, and recording the values of the output parameters (peak pressures and peak scaled impulses) along the length of the street at the pressure measuring points. The developed dataset was collected and formatted to 
conform to the Air3D model input and output parameters identified at the design stage. Afterwards, the neural network was trained on the data and further tested on new datasets not used in training to ensure its generalisation performance.

NeuroShell 2 [8], Release 4.0, was the neural network software utilised in the present study to train the network. The program implements several types of neural networks and architectures, including backpropagation nets, the multi-layer backpropagation nets, the genetic adaptive general regression neural networks (GRNN), the polynomial nets (GMDH), the backpropagation nets with jump connections and some others.

\subsection{Performance and analysis of the ANN models}

Four neural networks with different architectures were trained. Processing the actual data through the trained neural network produces the network's predictions for each pattern in the data file. Statistical analysis of the ANN models is given in Table 1 . Table 1 shows the $R^{2}$ (the coefficient of multiple determination) and the maximum absolute error (the maximum of |actual predicted| of all patterns). It can be seen that all three tested backpropagation networks demonstrated fairly good accuracy of the predicted overpressures and impulses with the coefficients of multiple determination being very close to 1 . The general regression neural network (GRNN) produced relatively low value of $R^{2}(0.794)$ for the predicted overpressures compared to the backpropagation networks $(0.993)$. The same tendency is observed with regard to the maximum absolute error introduced by the selected networks. The maximum absolute errors for both the overpressures and impulses predicted by GRNN are indicative of higher errors and lower accuracy of the results. It can be concluded from the analysis of the performance of the trial networks that the 4-layer backpropagation network configuration produced the most accurate predictions.

Table 1: $\quad$ Configuration and statistical performance of trial networks.

\begin{tabular}{|c|c|c|c|c|c|c|}
\hline \multirow[b]{2}{*}{$\begin{array}{l}\mathrm{N} \\
\mathrm{O}\end{array}$} & \multirow{2}{*}{$\begin{array}{c}\text { Network } \\
\text { Architecture }\end{array}$} & \multicolumn{3}{|c|}{ Configuration of networks } & \multicolumn{2}{|c|}{$R^{2}$} \\
\hline & & $\begin{array}{l}\text { Input } \\
\text { units }\end{array}$ & $\begin{array}{l}\text { Hidden } \\
\text { units }\end{array}$ & $\begin{array}{c}\text { Output } \\
\text { units }\end{array}$ & Pressure & Impulse \\
\hline 1 & $\begin{array}{c}\text { 3-layer } \\
\text { backpropagation }\end{array}$ & 3 & 28 & 2 & 0.975 & 0.982 \\
\hline 2 & $\begin{array}{c}\text { 4-layer } \\
\text { backpropagation }\end{array}$ & 3 & $14 / 14$ & 2 & 0.993 & 0.995 \\
\hline 3 & $\begin{array}{c}\text { 5-layer } \\
\text { backpropagation }\end{array}$ & 3 & $8 / 8 / 8$ & 2 & 0.993 & 0.995 \\
\hline 4 & $\begin{array}{l}\text { GRNN (general } \\
\text { regression NN) }\end{array}$ & 3 & 1892 & 2 & 0.794 & 0.967 \\
\hline
\end{tabular}

\section{Results and discussion}

As discussed earlier, the database of blast effects was generated using the blast propagation code Air3D for a typical straight street configuration. Several trial 
neural networks were tested and the best network with least absolute error in both output variables was selected. The 4-layer backpropagation neural network with 2 hidden layers with eight units in each hidden layer was found to produce the best predictions for the blast effects across all ranges of the input parameters.

The trained neural network was used to estimate the blast peak overpressure and peak impulse for a range of input parameters used to generate the database of blast effects from a series of CFD numerical simulations. Figure 3 demonstrates the comparison of the peak pressures and impulses along a straight street predicted by the two methodologies for the value of the scaled street width representing narrow city streets. From Figure 3, it can be seen that the blast effects predicted by the trained neural network show very good agreement with those obtained from the CFD simulations. In particular, the positive impulses predicted by the trained neural network are in very close agreement with the CFD generated values. Based on the obtained results, it has been proven that a neural network can be used as an effective tool for rapid prediction of blast effects in urban environments.

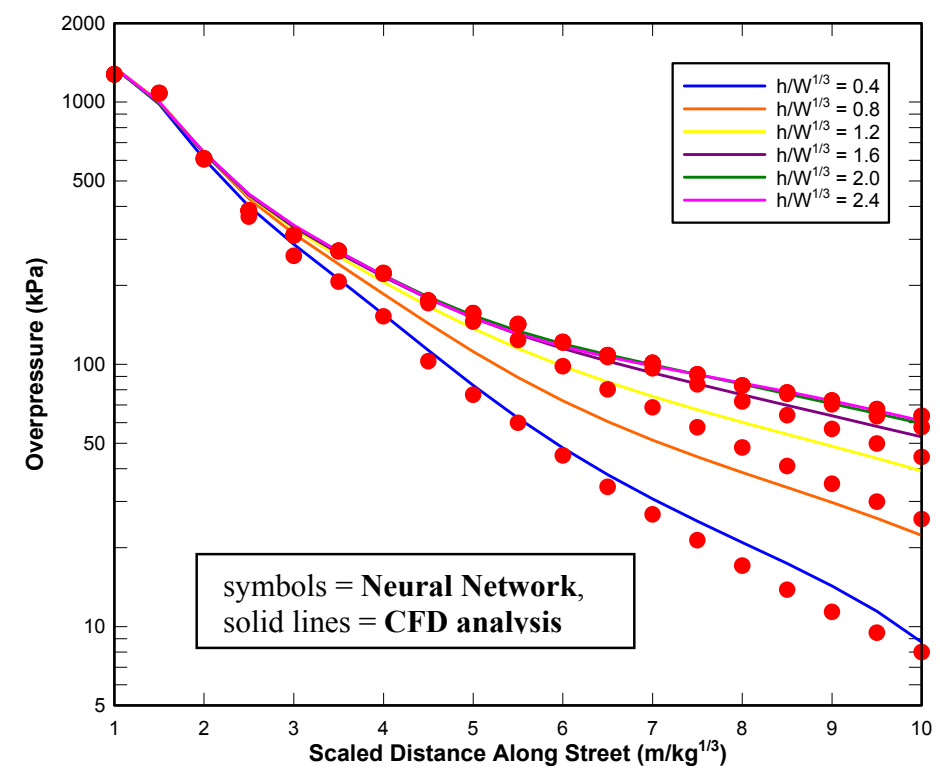

Figure 3: Comparison of CFD- and neural network-based blast overpressures and impulses along the street (scaled street width $=0.8 \mathrm{~m} / \mathrm{kg}^{1 / 3}$ ).

\section{Conclusions}

The main objective of this study was to evaluate a new approach of using artificial neural network for predicting the blast loads in complex city environments. A database of blast effects was built from a series of CFD blast simulations for a selected street configuration. This database was used to train 
and test the neural network. Analysis of the network's performance has demonstrated that the neural network can replace the time-consuming CFD analyses for a given street configuration and within the boundaries of the existing database of blast effects for this street configuration. It is expected to utilise the developed neural network-based technique as a part of an expert system that would be capable of predicting information about the likely injury and damage levels should an explosion occurs in a variety of urban environments based on the fast running predictive models.

\section{References}

[1] Century Dynamics, AUTODYN-2D \& $3 \mathrm{D}$ Version 6.0 User Documentation, 2005.

[2] Rose TA. Air3D User's Guide. 7.0: RMCS, Cranfield University, UK; 2003.

[3] Dayhoff JE. Neural network architectures: an introduction. New York: Van Nostrand Reinhold, 1990.

[4] Rose TA, Smith PD., Influence of the principal geometrical parameters of straight city streets on positive and negative phase blast wave impulses. International Journal of Impact Engineering, 27, pp. 359-376, 2002.

[5] Remennikov, A.M., Evaluation of blast loads on buildings in urban environment". Proc. of the $8^{\text {th }}$ International Conference on Structures Under Shock and Impact, Greece, pp. 73-82, 2004.

[6] Remennikov, A.M., "Blast effects analysis for commercial buildings in urban setting". Proc. of the $18^{\text {th }}$ Australasian Conference on the Mechanics of Structures and Materials (ACMSM), Perth, pp. 969 - 974, 2004.

[7] Remennikov, A.M. and Rose, T.A., Modelling blast loads on buildings in complex city geometries, International Journal of Computers and Structures, 83, pp. 2197-2205, 2005.

[8] NeuroShell 2, Release 4.0, 2000. User's manual. Ward Systems Group Inc., Frederick, MD. 